\title{
Metalización Superficial de Materiales no Metálicos
}

RESPONSABLE: Ing. Freddy Cori Nina

RESUMEN Se ha investigado la influencia de la concentración del $\mathrm{CuSO}_{4}-5 \mathrm{H}_{2} \mathrm{O}$ de la solución de premetalizado y el tiempo de tratamiento sobre el espesor de la capa depositada en la superficie de las muestras de polietileno de alta densidad (PEAD). En la primera parte se realizó un premetalizado por inmersión en la solución correspondiente, el método es autocatalítico, siendo su objetivo proporcionarle conductividad superficial a las muestras de plástico.

En la segunda parte se trata re reforzar la capa premetalizada por el método electroquimico en una solución ácida de sulfato de cobre. En la tercera parte es de aplicarle, a las muestras de polietileno, el metalizado con soluciones de cromo y por método electroquímico, con el fin de conferirles buena apariencia y resistencia al desgaste y dureza. En el presente trabajo, los recubrimientos de los espesores de la capa de premetalizada fueron analizados con el microscopio metalográfico Metavar y, finalmente, evaluados con el diseño experimental, dando como resultado que de 3 - $4 \mathrm{~g}$ de sulfato de cobrey un tiempo de 15 - 24 minutos de tratamiento, se obtuvo un espesor homogéneo y muyaceptable.
MIEMBros: Ing. Carlos Gutiérrez Delgado.
INTRODUCCIÓN. El recubrimiento de una pieza de plástico con metal genera valor agregado, ya que no solo mejora la apariencia, sino que combina ligereza y flexibilidad de fabricar piezas de geometría compleja, así como resistencia al desgaste de los plásticos con las características superficiales y dureza de los metales. También los recubrimientos pueden aportar otras propiedades de gran importancia para aplicaciones específicas como pueden ser: disminuir el coeficiente de fricción, aumentar la resistencia, introducir propiedades ópticas especiales en la superficie o también producir colores y texturas con finalidad decorativa. Entre los productos que utilizan el metalizado de plástico están los envases de dulces y alimentos envueltos en bolsas de plástico aluminizado, que disminuyen el deterioro causado por la luz. Los aparatos electrodomésticos incluyen piezas plásticas metalizadas generalmente cromadas, tales como botones, interruptores y controles. Lo mismo ocurre con artículos de escritorio y juguetes diversos, también pueden ser utilizados en joyería de fantasía, artesanía como el recubrimiento de cerámicos. Los CD-ROM, discos compactos, minidiscos, videodiscos y quipos de sonido son ejemplos de soportes en los que una película metálica de aluminio

\begin{abstract}
We have investigated the influence of the concentration of $\mathrm{CuSO} 4-5 \mathrm{H} 2 \mathrm{O}$ and premetalizado solution treatment time, about the thickness of the layer deposited on the surface of the samples of high density polyethylene (HDPE). In the first part was performed by immersion in premetalizado corresponding solution, the method is autocatalytic, being its goal to provide surface conductivity to the plastic samples. In the second part is about strengthening re premetalizada layer by the electrochemical method in an acidic solution of copper sulfate. In the third part, is applicable to samples of polyethylene, metallized with chromium solutions by electrochemical method, to give them good looks and wear resistance and hardness. In this paper, the coating of the layer thicknesses were analyzed using premetalizada metallographic microscope Metaver and finally evaluated with the experimental design resulting from that of 3 to $4 \mathrm{~g}$ of copper sulfate and a time of 15 - 24 minutes of treatment, we obtained a uniform thickness and very acceptable.
\end{abstract}

se deposita sobre un sustrato plástico que la protege del deterioro ambiental. Estas películas, sin embargo, no se depositan por métodos electroquímicos, sino por medios físicos; pero es factible también obtener el mismo resultado por galvanoplastia. El método óptimo es evaluar desde el punto de vista de costos. Los recubrimientos con metales también pueden aplicarse sobre otros materiales no conductores como la madera, la cerámica, algunas piezas de autopartes y otros. La Facultad de Ingeniería Metalúrgica, en el sector de electroquímica y recubrimientos metálicos, cuenta con experiencia en las prácticas de laboratorio de los cursos anteriormente mencionados y con equipos convenientes que ha sido importante para el desarrollo del presente trabajo.

Galvanoplastía. Consiste en la deposición electrolítica de capas metálicas sobre materiales no metálicos, principalmente plásticos revestidos previamente de capas conductoras o sobre matrices negativas que posteriormente se separan las capas metálicas. La galvanoplastia utiliza los principios de la electroquímica. Las características del proceso son: 
Celda electroquímica. Se forma cuando 2 piezas de metal se ponen en contacto a través de un liquido conductor o electrolito. El circuito eléctrico completo que se produce permite tanto la electrodeposición como la corrosión electroquímica.

Componentes. La celda electroquímica comprende:

Elánodo: cede electrones al circuito y se corroe.

El cátodo: recibe electrones del circuito a través de una reacción química.

Contacto físico: el ánodo y el cátodo deben estar conectados eléctricamente por contacto físico, para que los electrones fluyan del ánodo al cátodo.

Electrolito: es el liquido que debe estar en contacto, tanto con el ánodo como con el cátodo. El electrolito es conductor por lo que completa el circuito.

Reacciones en la celda electroquímica. Una característica de los átomos metálicos es la pérdida o ganancia de electrones en las reacciones de oxidación o reacción anódica y la reducción o reacción catódica.

Reacción anódica o de oxidación. El ánodo (metal) experimenta una reacción por oxidación mediante la cual se ionizan (disuelven ) los atomos metálicos. Estos ingresan a la solución electrolitica, mientras que los electrones abandonan el ánodo a través de la conexión eléctrica:

$$
\mathrm{M} \rightarrow \mathrm{M}^{\mathrm{n}+}+\mathrm{ne}
$$

Metal sólido ión (o disuelto) M pierde sus ( $\mathrm{n}$ e ) electrones.

$$
\mathrm{Fe} \rightarrow \mathrm{Fe}^{2+}+2 \mathrm{e}^{-}
$$

La oxidación tiene lugar en el ánodo. Debido a que los átomos metálicos salen del ánodo este se corroe. Ejemplo.

$$
\mathrm{Al} \rightarrow \mathrm{Al}^{3+}+3 \mathrm{e}^{-}
$$

Reacción catódica o de reducción. Este proceso ocurre en el cátodo, lo cual es inverso de la reacción en el ánodo:

$$
\begin{aligned}
& \mathrm{M}^{\mathrm{n}+}+\mathrm{ne} \rightarrow \mathrm{M} \\
& \mathrm{Cu}^{\mathrm{n+}}+2 \mathrm{e}^{-} \rightarrow \mathrm{Cu}
\end{aligned}
$$

Los iones metálicos, tanto los añadidos intencionalmente al electrolito como los formados en la reacción del ánodo, se combinan con los electrones del cátodo.

Los plásticos. Son sustancias de origen orgánico formadas por largas cadenas macromoleculares, que contienen en su estructura carbono e hidrógeno principalmente. Se obtienen mediante reacciones químicas entre diferentes materias primas de origen sintético o natural. Es posible moldearlos mediante procesos de transformación aplicando calor y presión. Los plásticos son parte de la gran familia de los polímeros.

\section{MATERIALESY MÉTODOS}

\section{Materiales}

Se ha utilizado como muestra un polietileno de alta densidad ( PEAD ); este es un termoplástico fabricado a partir del etileno (elaborado a partir del etano, uno de los componentes del gas natural). Es muy versátil y se puede transformar de diversas formas: Inyección, Soplado, Extrusión o Rotomoldeo. Sus propiedades

\begin{tabular}{|c|c|}
\hline $\begin{array}{l}\text { Indice de fluider (MF, } 190 \mathrm{C} / 5 \mathrm{Kg} \text { ) Noma ISO } 1133 \text { - ASTM D } \\
1238\end{array}$ & $40.8 \mathrm{~g} / 10 \mathrm{~min}$ \\
\hline Densidad (Norma IS0 II83) & $>0.935 \mathrm{~g} / \mathrm{cm}^{7}$ \\
\hline $\begin{array}{l}\text { Resistencia a la tracción (Norma ISO R } 527 \text { - ISO } 6259 \text { ASTM D } \\
638 \text { ) }\end{array}$ & $>300 \mathrm{Kgem}^{2}$ \\
\hline Elongación a la rotura (Noma ISO R 527 - ISO 6259 ASTM D 638) & $>625 \%$ \\
\hline $\begin{array}{l}\text { Resistencia a la Presión intema (Norma IS0 1167- ISO 4427) s } \\
\text { ensayo }\end{array}$ & $\begin{array}{l}>90 \mathrm{Kg}^{\prime} \mathrm{cm}^{2}, 100 \\
\text { horas, a } 200^{\circ} \mathrm{C}\end{array}$ \\
\hline Impacto bod (Norma ASTM D 256) & $>13.6 \mathrm{~J} / \mathrm{m}$ \\
\hline
\end{tabular}
físico-químicas son: $\mathrm{Mw}=54.000 \mathrm{~g} / \mathrm{mol}, \mathrm{Mn}=19.000$ $\mathrm{g} / \mathrm{mol}$, temperatura pico de fusión $(\mathrm{Tm})$ de $134^{\circ} \mathrm{C}$ y grado de cristalinidad (XC) de $77 \%$.

Tabla 1: Características mecánicas y fisicas del polietileno.

Fuente: Gómez,J.L, (1998), Revista de Plásticos Modernos.

\section{Reactivos principales}

Los reactivos elementales son:

$\mathrm{Na}_{2} \mathrm{CO}_{3} ; \quad \mathrm{NaOH} ;$ detergente; $\mathrm{K}_{2} \mathrm{Cr}_{2} \mathrm{O}_{7} ; \mathrm{H}_{2} \mathrm{SO}_{4}$ concentrado; $\mathrm{H}_{2} \mathrm{O}$ destilada; $\mathrm{ClSn}_{2} ; \mathrm{HCl}$ concentrado; $\mathrm{PdCl}_{2} ; \mathrm{HCl} 0,01 \mathrm{M} ; \mathrm{CuSO}_{4}-5 \mathrm{H}_{2} \mathrm{O} ; \mathrm{NiCl}_{2}-6 \mathrm{H}_{2} \mathrm{O}$; formaldehído sin concentrar; Sal de Rechelle (tartrato doble de $\mathrm{Na}$ y $\mathrm{K}$; $\mathrm{Na}_{2} \mathrm{CO}_{3} ; \mathrm{NaaOH}$

\section{Equipos necesarios}

Un rectificador por medio del cual pueda controlarse la corriente y el voltaje, caimanes, termómetro, parrilla eléctrica, varios vasos de precipitado, una mufla u otros recipientes similares. Microscopio metalográfico Metavar. Equipos de preparación y pulido.

\section{Metodología}

Consistió en recubrir 10 muestras de plástico de polietileno de alta densidad (PEAD), previamente preparadas $15 \times 15 \times 5 \mathrm{~mm}$. Etapas del proceso, Figura 1 Etapas del metalizado de plásticos; el esquema del proceso de metalizado de las muestras de plásticos de polietileno del presente trabajo comprende las etapas siguientes: 
Figura 1. Diagrama de flujo del proceso de metalizado del polietileno

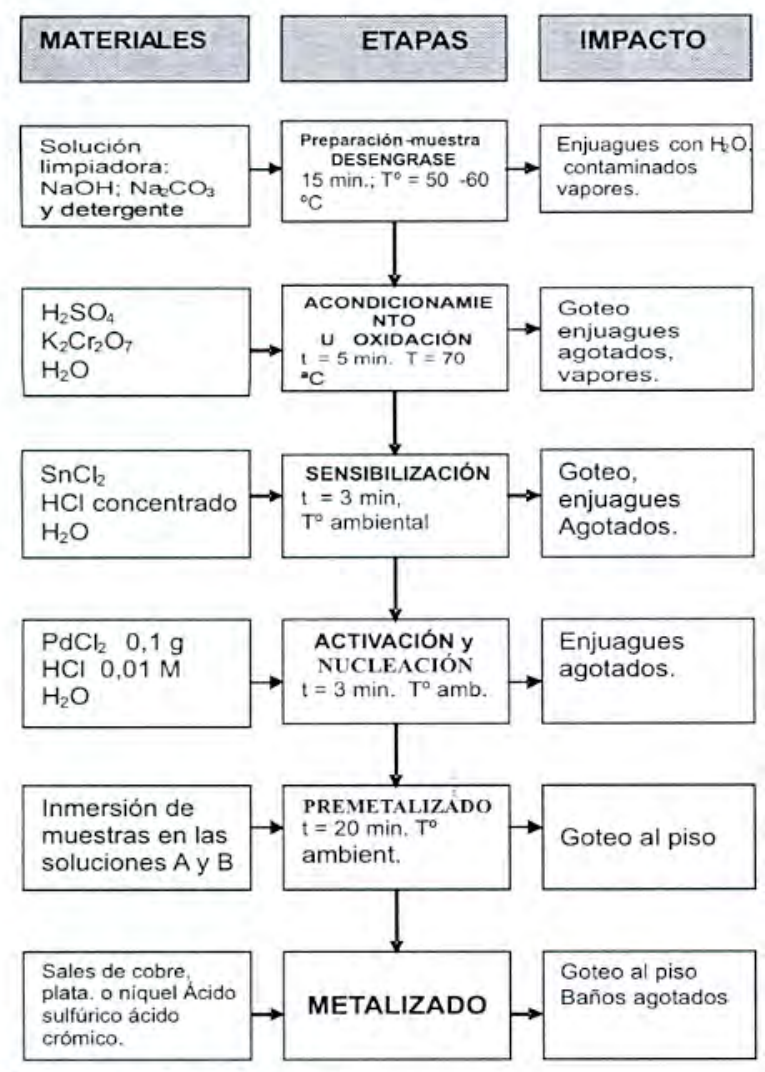

Fuente: Elaboración propia

El modelo elegido para el análisis de datos es el diseño experimental, donde a partir de los resultados de experiencias del laboratorio y el diseño factorial propuesto a dos niveles $2^{k}$ con 3 réplicas centrales del Statgraphics 5,1 , se obtendrá la ecuación posible que determine la capa óptima de recubrimiento de las muestras de plástico.

\section{RESULTADOSYDISCUSIÓN}

\section{Preparación de las probetas para el inicio del tratamiento}

Con las 10 probetas de plástico tipo polietileno, debidamente dimensionadas $15 \times 15 \times 5$ y pulidas adecuadamente se procedió a la preparación previa siguiente:

\section{Desengrase}

Se procedió a la limpieza, pulido y desengrase, ya que además, la mayoría de los plásticos tienen naturaleza hidrofóbica. Fue conveniente utilizar solventes. Aquí se produce un ligero ablandamiento superficial debido a la solventación parcial de las moléculas poliméricas y a una reacción parcial con el polímero. Los solventes utilizados son: La solución limpiadora, tiene la composición siguiente:

$$
\mathrm{Na}_{2} \mathrm{CO}_{3}=20 \quad \mathrm{~g} ; \mathrm{NaOH}=5 \quad \mathrm{~g} ; \text { detergente }=2 \mathrm{~g} ;
$$

Aforar con agua hasta $1000 \mathrm{ml}$.

Previamente, la solución se calentó de 50 a $60{ }^{\circ} \mathrm{C}$, luego por inmersión de las probetas de plástico se tomó un tiempo de exposición de 15 minutos. Finalmente se enjuagó con agua corriente, agua destilada y alcohol. Es importante tener cuidado de los vapores contaminados.

\section{Acondicionamiento u oxidación}

Se preparó la solución oxidante en un vaso de precipitado de $500 \mathrm{ml}$ :

Solución oxidante: $\mathrm{K}_{2} \mathrm{Cr}_{2} \mathrm{O}_{7}=50 \mathrm{~g} ; \mathrm{H}_{2} \mathrm{SO}_{4}$ conc. $=50 \mathrm{ml}$; $\mathrm{H}_{2} \mathrm{O}=120 \mathrm{ml}$.

Se calentó la solución a una temperatura de $70^{\circ} \mathrm{C}$ y con un tiempo de exposición de las muestras de 5 minutos. El acondicionamiento se realizó con la finalidad de proporcionar cierta rugocidad superficial, así como suministrar puntos de enlace químico para los metales aplicados posteriormente.

\section{Sensibilización y activación de la superficie}

Luego de prepararse la solución en la solución sensibilizadota siguiente:

Solución sensibilizadota: $\mathrm{ClSn}_{2}=60 \mathrm{~g} ; \mathrm{HCl}$ conc. $=60 \mathrm{ml}$. Luego se han sumergido las muestras en la solución a temperatura ambiente y un tiempo de exposición por 3 minutos. Se enjuagan luego las muestras con agua corriente y agua destilada. La sensibilización consiste en la adsorción, en la superficie del plástico, de un material fácilmente oxidable.

\section{Activación y nucleación}

Para la activación se preparó la siguiente solución:

Solución activadora: $\mathrm{PdCl}_{2}=0,1 \mathrm{~g} ; \mathrm{HCl} 0,01 \mathrm{M}=200 \mathrm{ml}$. Se colocan las muestras en la solución a temperatura ambiente y durante 3 minutos; luego se lavan con agua corriente y agua destilada. La oxidación en la etapa de nucleación sirve para depositar una superficie catalítica en la superficie plástica.

\section{Premetalizado}

El premetalizado es un proceso autocatalítico; se logra sumergiendo lasmuestras, en una mezcla a partes iguales de las soluciones A y B que contienen cobre. Esta operación se realiza sin uso de corriente eléctrica, el objetivo aquí es lograr una superficie suficientemente conductora para el posterior proceso electrolítico. Las soluciones para el premetalizado son: 
Solución A

$\begin{array}{lcl}\mathrm{CuSO}_{4}-5 \mathrm{H}_{2} \mathrm{O} & 14 & \mathrm{~g} \\ \mathrm{NiCl}_{2}-6 \mathrm{H}_{2} \mathrm{O} & 4 & \mathrm{~g} \\ \begin{array}{l}\text { Formaldehído sin concentrar } \\ \text { Se disuelve en } 1000 \mathrm{ml} \text { de agua destilada }\end{array} & 52 & \mathrm{ml} \\ & & \\ \text { Solución B } & & \\ \text { Tartrato doble deNa y K } & 45 & \mathrm{~g} \\ \mathrm{Na}_{2} \mathrm{CO}_{3} & 4 & \mathrm{~g} \\ \mathrm{NaOH} & 11 & \mathrm{~g} \text { ó hasta }\end{array}$

conseguir $11-12 \mathrm{pH}$. Y se disuelve en $1000 \mathrm{ml}$ de agua destilada.

\section{Metalizado intermedio}

Es un proceso electrolítico; es la segunda capa con el fin de reforzar el premetalizado, con soluciones ácidas de $\mathrm{CuSO}_{4}-\mathrm{H}_{2} \mathrm{O}$. Se utiliza baja densidad de corriente de unos $0,5 \mathrm{~A} / \mathrm{dm}^{2}$ y a temperatura ambiente. Se inicia con el depósito de 15 ìm de cobre, en un baño de sulfato de cobre. La ASTM sugiere que el espesor óptimo de la capa de metal sea entre 13 a 20 im. Las características del electrolito son $40 \mathrm{~g} / 1$ de sulfato de cobre, actuando de cátodo las muestras de polietileno y de ánodo acero inoxidable.

\section{Metalizado o electrorrecubrimiento.}

El recubrimiento final se realiza con cromo para fines decorativos o de resistencia al desgaste y a la corrosión en ambientes moderados. Los recubrimientos de cromo decorativo son catódicos. El mecanismo de la reacción según Zinder y Jones en 2004, para la deposición del cromo, es el siguiente:

Reacción de deposición de cromo

$2 \mathrm{CrO}_{3}+2 \mathrm{H}_{2} \mathrm{O} \rightarrow 2 \mathrm{H}_{2} \mathrm{CrO}_{4}$

$2 \mathrm{H}_{2} \mathrm{CrO}_{4} \rightarrow \mathrm{Cr}_{2} \mathrm{O}_{7}^{-2}+\mathrm{H}_{2} \mathrm{O}+2 \mathrm{H}^{+}$

$\mathrm{Cr}_{2} \mathrm{O}_{7}{ }^{-2}+14 \mathrm{H}^{+}+12 e^{-}$(catalizador $) \rightarrow 2 \mathrm{Cr}^{0}+7 \cdot \mathrm{H}_{2} \mathrm{O} \ldots \ldots \ldots(8)$

Reacción colateral

$$
2 \mathrm{H}^{+}+2 e^{-} \rightarrow \mathrm{H}_{2 \text { gas }} \text {. }
$$

Una solución de ácido crómico, en un alto grado de saturación, se utiliza como electrolito. El cromo ha probado ser satisfactorio, para partes resistentes al desgaste, debido a su extrema dureza, que excede a la mayor parte de los otros metales comerciales.

\section{Resultados}

Espesor de la capa premetalizada.

Luego de concluirse el tratamiento de premetalizado, 5 probetas de polietileno previamente son preparadas, haciendo cortes transversales para ver el espesor de la capa premetalizada a través del microscopio metalográfico Metavar y los datos se muestran en la Tabla 2 .
Tabla 2 Matriz del diseño con valores reales parapruebas

\begin{tabular}{|c|c|c|c|c|}
\hline \multicolumn{2}{|c|}{$\mathbf{N}^{\circ}$ de prueba } & $\begin{array}{c}\mathrm{CuSO}_{4} \\
\mathbf{H}_{2} \mathbf{O} \\
\text { (g) }\end{array}$ & $\begin{array}{c}\text { Tiempo } \\
\text { (min.) }\end{array}$ & $\begin{array}{c}\text { Espesor } \\
\text { (mm) }\end{array}$ \\
\hline & I & $2(-)$ & $6(-)$ & 0,0081 \\
& II & $4(+)$ & $6(-)$ & 0,0101 \\
& III & $2(-)$ & $24(+)$ & 0,0093 \\
& IV & $4(+)$ & $24(+)$ & 0,0115 \\
\hline \multirow{2}{*}{ PRODUCTOS } & $\mathbf{V}$ & 3 & 15 & 0,0097 \\
CENTRALES & $\mathbf{V}$ & 3 & 15 & 0,0100 \\
\hline
\end{tabular}

Donde se concluye que la probeta de mayor relevancia se estima con el tratamiento a 3 - 4 gramos de $\mathbf{C u S O}_{4}-$ $\mathbf{5} \mathrm{H}_{2} \mathrm{O}$, proveniente de la solución de premetalizado $\mathrm{A}$ - B y durante un tratamiento de 15 - 24 minutos, obteniéndose un espesor de $0,0100 \mathrm{~mm}$. La ecuación del modelo de la regresión que se ajusta a los datos es la siguiente:

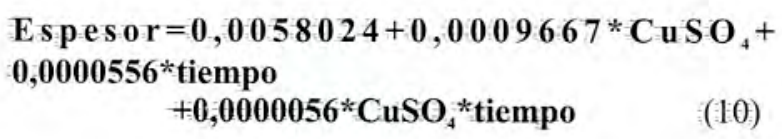

Donde:

$\begin{array}{ll}\text { Constante } & =0,0058024 \\ \text { A: } \text { CuSO }_{4} & =0,0009667 \\ \text { B: Tiempo } & =0,0000556 \\ \text { AB: } & =0,0000056\end{array}$

Superficie respuesta delmatrialmetalizado.

El gráfico de Pareto visualiza la importancia relativa de los factores sobre el espesor. Fanto A y B muestran tener gran influencia en el proceso de metalización. De mayor preponderancia es el factor A, es decir, la mayor concentración de $\mathrm{CuSO}_{4}$ en la solución permite mejorar el proceso, como se muestra en la Figura 2.

Figura $\mathrm{N}^{\circ} 2$ Gráfico de Pareto

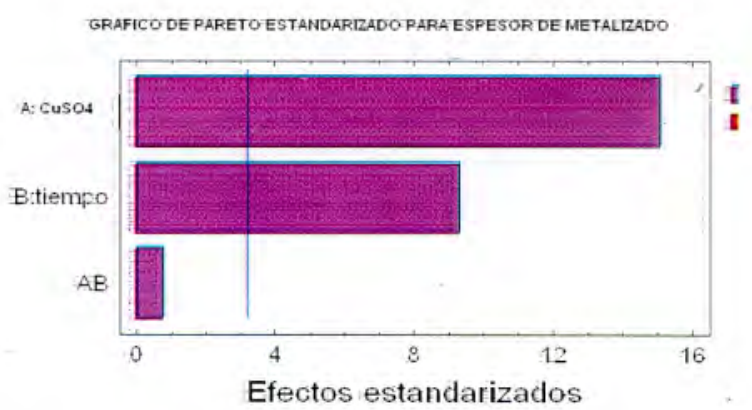

Curva de isovalores

La curva de isovalores muestra en dos dimensiones el comportamiento de las variables dependientes para obtenerse la variable respuesta en una columna al costado. 
Figura $\mathrm{N}^{\circ} 4$ Curva de isovalores para el espesor del recubrimiento.

\section{PREMETALIZADO}

\section{Contornos de Superficie de la Respuesta Estimada}

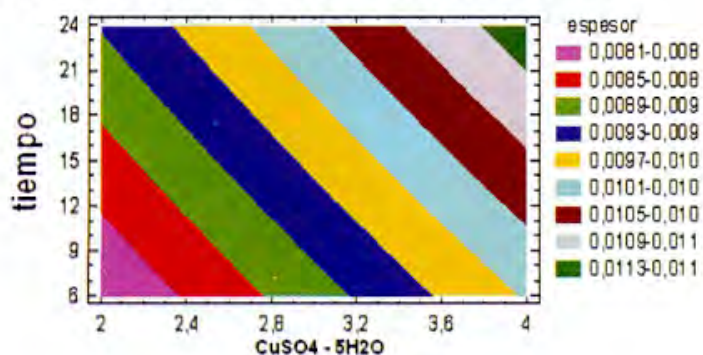

En la Figura 4 se aprecia la curva de isovalores, donde se notan diversos espesores para cada relación de factores de $\mathrm{CuSO}_{4}$ y el tiempo; aqui se puede distinguir que al tiempo de 24 minutos y 4 gramos $\mathrm{Cu}$, el espesor es óptimo entre 0,011 y $0,0113 \mathrm{~mm}$ de espesor de la capa; que son uno de los valores más altos.

\section{CONCLUSIONES}

1. El recubrimiento de una pieza de plástico como el palietileno de alta densidad (PEAD) con metal, genera valor agregado ya que no solo mejora la apariencia, sino que combina ligereza y flexibilidad de fabricar piezas de geometría compleja, así como resistencia al desgaste de los plásticos con las características superficiales y dureza de los metales.

2. Existe influencia de la concentración del $\mathrm{CuSO}_{4}-$ $5 \mathrm{H}_{2} \mathrm{O}$ de la solución de premetalizado y el tiempo de tratamiento sobre el espesor de la capa depositada en la superficie de las muestras de polietileno.

3. El presente trabajo se realizó en tres partes: primero el premetalizado, en un proceso autocatalítico con la finalidad de conferirle a la superficie de la muestra conductividad eléctrica. La segunda parte metalizado medio, por el proceso electroquímico con la finalidad de reforzar la capa premetalizada. Y la tercera parte, recubrimiento electroquímico en soluciones sobre la base del cromo con la finalidad de darle buena apariencia y reistencia al desgaste.

4. Los recubrimientos de los espesores de la capa premetalizada fueron analizados con el microscopio metalográfico Metavar y finalmente evaluados con el diseño experimental; dando como resultado de que de 3 - $4 \mathrm{~g}$ de sulfato de cobre y un tiempo de 15 24 minutos de tratamiento, se obtuvo un espesor homogéneo y muy aceptable.

\section{REFERENCIAS BIBLIOGRÁFICAS}

ASKELAND, D. Ciencia e ingeniería de los materiales. $3^{\circ} \mathrm{ed}$. Ed. InternacionalT homson, 1998.

BOWDEN, F., Tabor, D. The friction and lubrication of solids. Part II. Oxford, Clarendon press, 1964.

CALLISTER, W. Introducción a la ciencia e ingeniería de los materiales. Tomo II. Editorial Reverté, Barcelona, 1996.

PÉREZ, C. Estudio del desgaste por deslizamiento en seco en un equipo "Pin-ondisk". Trabajo Dirigido de Grado. Universidad Nacional de Colombia, Sede Medellín, 1999.

Plásticos Industriales. Catálogo de productos. Smith, W. Fundamentos de la ciencia e ingeniería de los materiales. Ed. McGraw Hill Book Company, 1993.

AVNER S. (1991), Introducción a la metalurgia física. Editorial Mc.Graw Hill, México, 317-325 pp.

DONALD R. ASKELAND, La ciencia e ingeniería de los materiales. Grupo Editorial Iberoamericana, México, 1987; 110,498-499. 
ANEXOS

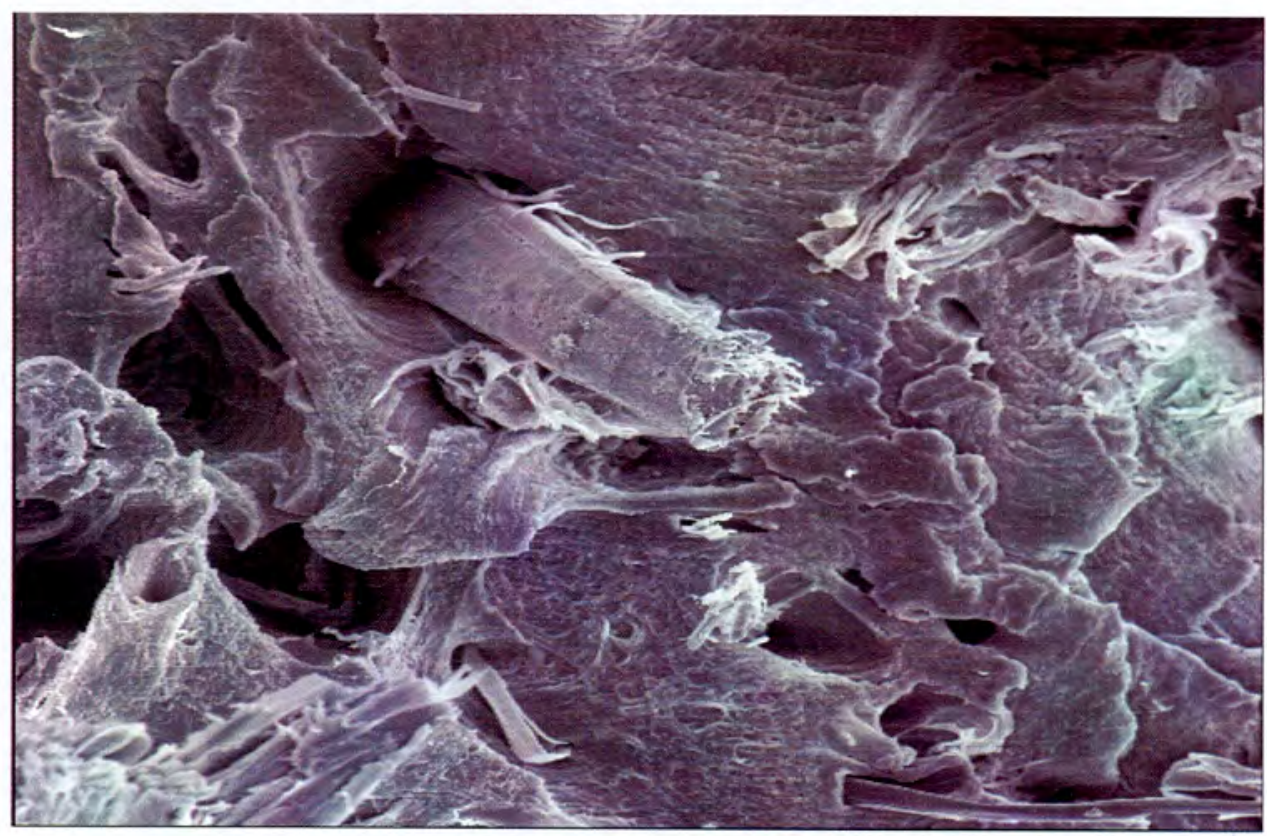

Fotografia con el microscopio electrónico de barrido de la sección de rotura de una probeta a tracción, de polipropileno

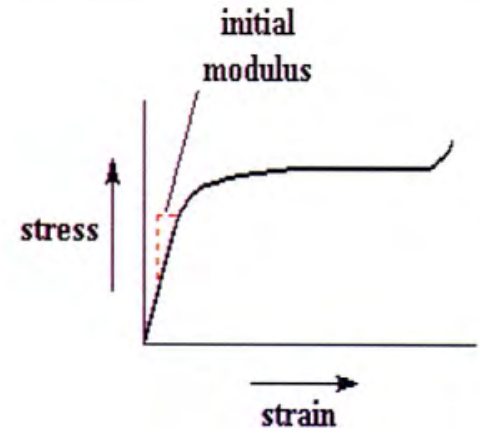

Variación de la curva tensión - estiramiento.

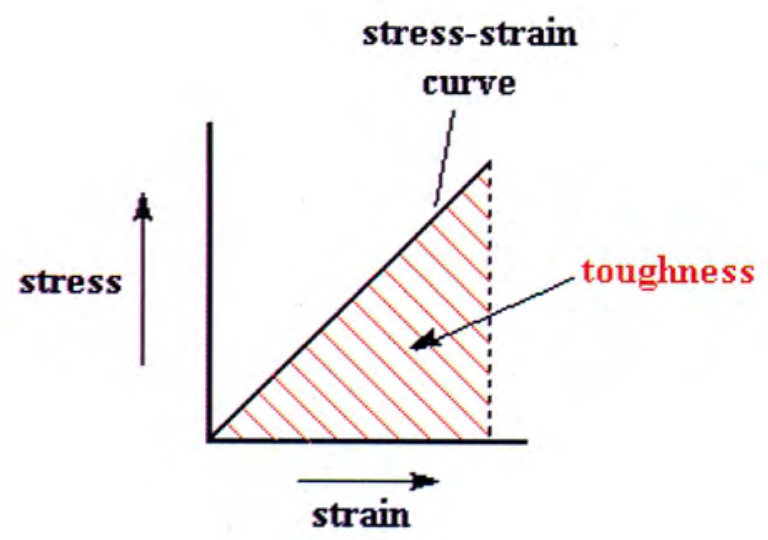

Variación de tensión- deformación que muestra la dureza.
Superficie de Respuesta estimada

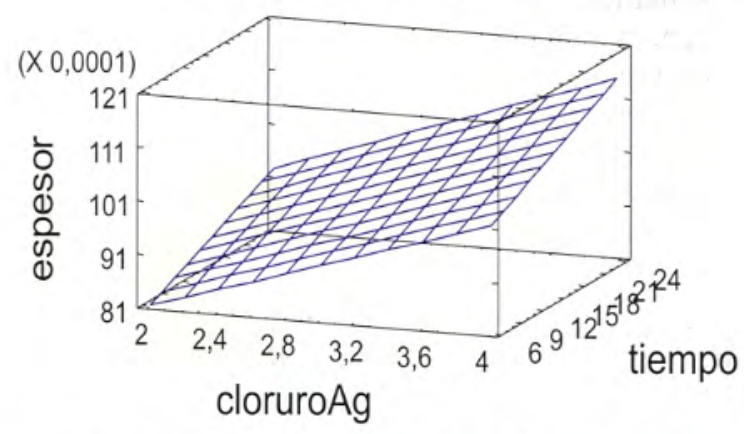

Superficie respuesta de los datos estimados del metalizado

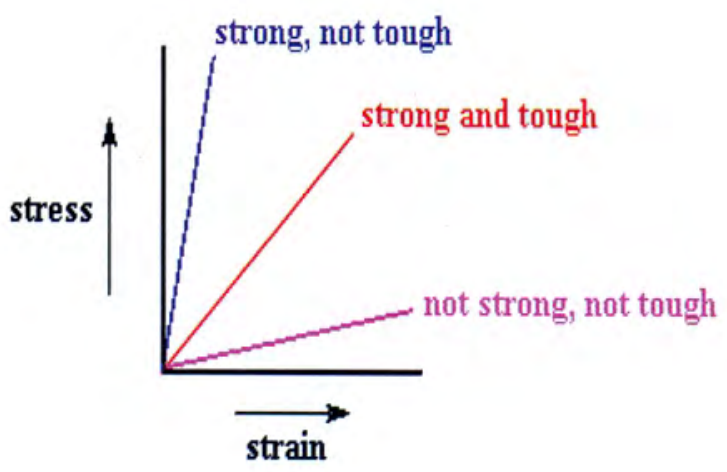

Relación tensión - estiramiento que compara diferentes tipos. de plástico. 